\title{
Relation of elevated serum ferritin levels to hypothyroidism in children with beta-thalassemia major
}

\author{
Kundu D $D^{1}$, Ray $D^{2}$, Ghosh $S^{3}$, Chowdhury DG ${ }^{4}$, Dutta $S^{5}$, Dhar $A^{6}$ \\ ${ }^{1}$ Dr. Dipankar Kundu, Associate Professor, ${ }^{2}$ Dr. Debes Ray, Associate Professor, ${ }^{3}$ Dr. Srinika Ghosh, BSc (Hons), MSc \\ (Medical Biochemistry), Final Year Student, ${ }^{4}$ Dr. Debkanya Gon Chowdhury, BSc (Hons), MSc (Medical Biochemistry), \\ Final Year Student, ${ }^{5}$ Dr. Suparna Dutta, BSc (Hons), MSc (Medical Biochemistry), Final Year Student, ${ }^{6}$ Dr. Aninda \\ Dhar, BSc (Hons), MSc (Medical Biochemistry), Final Year Student. All are affiliated with Department of Biochemistry, \\ Medical College, Kolkata, West Bengal, India
}

Address for Correspondence: Dr. Dipankar Kundu, Associate Professor, 12Q/1F, Paikpara, Kolkata-37, West Bengal, India. Email: dr.dipankar@yahoo.co.in

\begin{abstract}
Background: The commonest form of thyroid dysfunction, seen in thalassaemics, is subclinical hypothyroidism due to abnormalities of the thyroid gland which, leads to insufficient production of thyroid hormones. However, the frequency of hypothyroidism varies depending on the region, quality of management and treatment protocols. Aim: This study was conducted with the aim of investigating the frequency of hypothyroidism in children with beta-thalassemia major and to study its correlation with serum ferritin. Material and methods: A case-control study was carried out in the OPD, Medical College, Kolkata. It included 100 children with Beta-Thalassemia major as cases. Hundred age and gender matched healthy controls were also included in the study. Results: The weight and height of the cases lagged behind those of the control group. Compared to the control group, the mean level of thyroid hormones (both T3 and T4) and Hemoglobin levels were significantly reduced while those of TSH and Serum Ferritin were significantly increased. Out of 100 cases, 64 were euthyroid, 34 and 2\% had subclinical and clinical hypothyroidism respectively. No case of secondary hypothyroidism was detected. Our results showed no association between Serum ferritin levels and the frequency of hypothyroidism among studied patients. Conclusion: Thyroid dysfunction in thalassemia may start early in life, hypothyroidism is not clinically observed in most thalassemia major patients. Therefore, thyroid function should be followed on regular basis when other iron over load associated complications occurs. Regular follow-up for early detection and timely treatment of such complications could improve the quality of life of these patients.
\end{abstract}

Key words: $\beta$-thalassemia, thyroid profile, ferritin, hypothyroidism.

\section{Introduction}

Hypothyroidism usually appears in the second decade of life and is thought to be associated with iron overload in patients with thalassemia major [1]. Beta-thalassemia represents a group of recessively inherited hemoglobin disorders characterized by deficient synthesis of the $\beta$ globin chain.

Beta-thalassemia is one of the most common genetic diseases in India. Every year around 100000 children were born with thalassemia major in the world and

Manuscript received $14^{\text {th }}$ November 2016

Reviewed: $25^{\text {th }}$ November 2016

Author Corrected: $5^{\text {th }}$ December 2016

Accepted for Publication 20 $0^{\text {th }}$ December 2016 around 10000 were born in India alone. The carrier rate of $\beta$ thalassemia gene varies between $1-3 \%$ in south India and $5-15 \%$ in north India. The disease was previously considered fatal before 2 nd decade of life [2]. The homozygous state results in severe anemia in infancy, which requires regular blood transfusion.

The combination of transfusion therapy and chelation therapy has dramatically extended the life expectancy of the children with $\beta$ thalassemia major who can now live into their third and fourth decades. The only curative treatment available is stem cell transplant which is not affordable in countries like India [3]. 
Thalassemia has been studied in different areas of India by different researchers. However, the changes in the endocrine system of these patients have not yet been studied. These patients are dependent on blood transfusions to maintain the levels of hemoglobin and packed cell volume in their blood, but siderosis is a major complication of treatment. Repeated transfusions lead to accumulation of iron in different tissues, including the tissues of the endocrine glands.

These disorders have been proven to be the result of hemosiderosis of secretory cells such as the gonadotroph cells of the pituitary gland [4]. Most complications are caused by increased iron sedimentation in tissues like heart, endocrine glands and these results in heart failure, arrhythmia, hypothyroidism and diabetes mellitus. Thyroid dysfunction is known to occur frequently in thalassemia major, but its prevalence and severity varies in different cohorts and the long-term natural history is poorly described. Despite therapy with defroxamine to treat iron overload, the risk of secondary endocrine dysfunction remains high.

Hypogonadism was one of the most frequent endocrine complications seen in one study [5]. Impaired glucose tolerance, short stature, hypocalcaemia, and subclinical and overt hypothyroidism are also frequent [6].

Decrease production of thyroid hormones according to body demand or defect in thyroid hormone receptors cause hypothyroidism. In several studies, hypothyroidism has been reported to be correlated with serum ferritin level; although in some studies there were no such correlations. Contrarily to significant iron deposition in thyroid gland, low activity remains about subclinical hypothyroidism. Thyroid dysfunctions are well documented in patients with thalassemia major requiring frequent and recurrent blood transfusion. These have recently been discussed in details in the literatures $[4,5,6]$. Also, growth retardation is another complication that usually occurs. However, it almost will not happen with sequential transfusion. Nonetheless, defroxamine overuse causes growth retardation by itself [5].

Although many studies report endocrinopathy in thalassemic patients, results are controversial and different. Clinically overt manifestations of hypothyroidism occur late in life and most of the studies available are done on adults. Only a very few pediatric studies are available. In India, cost of chelation precludes ideal therapy for majority of the patients and the compliance with transfusion is often not optimal. Therefore there is a possibility that there may be high prevalence of hypothyroidism in thalassemic children. In view of very few pediatric Indian studies, we therefore planned the present study with the aim to assess thyroid function in children suffering from $\beta$ thalassemia major with iron overload and to evaluate its relation, if any, with serum ferritin levels [7]. We evaluated the pituitary/thyroid axis in thalassemia major patients in this cross sectional study and correlated T4 and TSH secretion with the ferritin level.

Aim: This study was conducted with the aim of investigating the frequency of hypothyroidism in children with beta-thalassemia major and to study its correlation with serum ferritin and transfusion index.

\section{Material and Methods}

A case-control study was carried out in the OPD, Medical College, Kolkata. It included 100 children with Beta-Thalassemia major (The diagnosis of thalassemia major was based on the usual hematological criteria i.e. peripheral blood evaluation and Hemoglobin electrophoresis) as cases. Hundred age and gender matched healthy controls were also included in the study. The institutional ethical committee approved the study protocol. Informed consent was taken from the parents of the cases and control subjects. Patients diagnosed as Beta thalassemia major, age ranged from 5-12 years of both genders having blood transfusion more than 2 years were included in the study. Other types of chronic hemolytic anemia, presence of other endocrinal disorders and terminally ill patients were excluded from this study. Hundred age and sex matched healthy children constituted the control group. Laboratory investigations included Serum ferritin T3 and T4, TSH by using CLIA [8].

Hypothyroidism was defined as TSH level $>6.4 \mu \mathrm{IU} / \mathrm{ml}$, $\mathrm{T} 4$ levels $<0.6 \mu \mathrm{g} / \mathrm{dl}$ and T3 levels $<2.4 \mu \mathrm{g} / \mathrm{dl}$. The thyroid function status of the patients was classified as compensated (increased TSH, normal T4, T3) and uncompensated (increased TSH, decreased T4 and/or T3) primary hypothyroidism, and euthyroidism (normal TSH, normal free T4) [9].

Statistical Analysis: Data was collected and double entered into Microsoft Access and data analysis was performed using SPSS 15.0 software version 18 under 
windows 7. Simple descriptive analysis in the form of numbers and percentages for qualitative data, and arithmetic means as central tendency measurement, standard deviations as measure of dispersion for quantitative parametric data, and inferential statistic test were used. ANOVA test in comparing more than two independent groups of quantitative data were used. For qualitative data, Chi square test to compare two of more than two qualitative groups and bivariate correlation test were used to test associations between variables. The level $p \leq 0.05$ was considered the cut-off value for significance.

\section{Results}

The two groups had no significant differences in age and gender. The weight and height of the cases lagged behind those of the control group. Compared to the control group, the mean level of thyroid hormones (both T3 and T4) and Hemoglobin levels were significantly reduced while that of TSH was increased significantly. Out of 50 cases, 32(64\%) were euthyroid, $17(34 \%)$ and $1(2 \%)$ had subclinical and clinical hypothyroidism respectively. No case of secondary hypothyroidism was detected.

Table-1: Demographic, hematological and biochemical characteristics of cases and controls.

\begin{tabular}{|c|c|c|c|}
\hline Characteristics & Cases & Controls & P value \\
\hline Age $(\mathrm{yrs})$ & $6.98 \pm 2.98$ & $6.62 \pm 2.70$ & $>0.05$ \\
\hline Male: Female & $56: 44$ & $52: 28$ & $>0.05$ \\
\hline Height (centimeter) & $114.09 \pm 17.29$ & $117.12 \pm 17.01$ & $<0.05$ \\
\hline Weight $(\mathrm{kgs})$ & $22.64 \pm 8.18$ & $24.10 \pm 7.87$ & $<0.05$ \\
\hline Hemoglobin $(\mathrm{gm} / \mathrm{dl})$ & $8.2 \pm 1.11$ & $11.9 \pm 1.01$ & $<0.05$ \\
\hline Serum Ferritin(ng/ml) & $2903.10 \pm 772.26$ & $86.10 \pm 24.78$ & $<0.05$ \\
\hline TSH $(\mu \mathrm{IU} / \mathrm{ml})$ & $7.15 \pm 8.92$ & $2.51 \pm 1.06$ & $<0.05$ \\
\hline T3 $(\mathrm{ng} / \mathrm{ml})$ & $1.12 \pm 0.39$ & $1.30 \pm 0.42$ & $<0.05$ \\
\hline T4 $(\mathrm{ng} / \mathrm{ml})$ & $95.11 \pm 16.97$ & $99.11 \pm 12.98$ & $<0.05$ \\
\hline
\end{tabular}

Table-2: Association between thyroid hormones and different parameters.

\begin{tabular}{|c|c|c|c|c|c|c|c|c|}
\hline & \multicolumn{2}{|c|}{ Age } & \multicolumn{2}{c|}{ Weight } & \multicolumn{2}{c|}{ Height } & \multicolumn{2}{c|}{ Ferritin } \\
\hline & r & p & r & P & r & p & r & p \\
\hline TSH & 0.08 & 0.65 & 0.02 & 0.90 & 0.11 & 0.87 & 0.08 & 0.31 \\
\hline T3 & 0.02 & 0.82 & 0.02 & 0.92 & 0.09 & 0.90 & 0.08 & 0.52 \\
\hline T4 & 0.01 & 0.61 & 0.22 & 0.37 & 0.08 & 0.42 & 0.40 & 0.26 \\
\hline
\end{tabular}

There was no significant correlation between T3, T4 and Serum Ferritin levels. Also there was no significant correlation between TSH level and Serum Ferritin.

\section{Discussion}

The two groups had no significant differences in age and gender. Compared to the control group, the studied patients had a lower mean height, weight and Hemoglobin levels; the causes for this are multiple and include endocrinal impairment, chronic anemia and social, nutritional reason, however, it is accepted that thalassemic patients have a delayed puberty and slower growth rate but ultimately the majority will catch up with their peers as age advances [10]. There was no correlation between thyroid dysfunction and gender and age. Similar results have been documented by Gathwala et al [11].
Endocrine dysfunction is the second most frequent complication, over $60 \%$ of thalassaemics after the age of 10 years have at least one endocrine gland dysfunction and about $40 \%$ have multiple endocrinopathies [12]. In our study, 34\% thalassemic children had subclinical hypothyroidism which is in good agreement with the study done by Sharma et al [13]. We also found that $2 \%$ thalassemic children had clinical hypothyroidism which was in agreement with Agrawal and Zervas et al [13]. The reason for the lower frequency may be attributed to the fact that the majority of patients in the present work were under 10 years old. 
Not many studies are available from India and one among the very few studies done by N. K. Anand revealed that $32 \%$ of patients had subclinical hypothyroidism and $12 \%$ had clinical hypothyroidism [14]. This finding is comparable to our study in terms of subclinical hypothyroidism. Hyper-transfusion has improved the life expectancy of thalassemic patients, over the decades. However, chelation therapy is expensive, difficult to administer and not as readily available, hence the compliance is often poor despite regular transfusions resulting in iron overload [7]. It has been demonstrated that thyroid abnormalities in these patients are related to iron overload. Histological studies have supported this hypothesis [15].

However, the serum ferritin is the most widely used test for assessment of iron status in these patients. Iron overload of tissue is the most important complication of beta-thalassemia and is a major subject of management [5]. Although most clinical signs of iron loading do not appear until the second decade of life in patients with inadequate chelation, evidence from serial liver biopsies in very young patients present that the toxic effects of iron begins much earlier. After approximately one year of transfusions, iron starts accumulating in parenchymal tissues, where it may bring about substantial toxicity as compared with that within reticulo-endothelial cells. Despite the reports relating endocrine dysfunction with iron overload, it was recently demonstrated that the degree of iron overload, at least reflected by ferritin levels, was not associated with the development of endocrine complications [8]. In this study, a significant association was found between ferritin levels and thyroid functional status; the ferritin levels of hypothyroid patients being significantly higher than euthyroid patients.

The precise mechanism by which iron overload causes tissue damage is not completely understood, though it is suggested that tissue iron deposits act at the cellular level causing damage via free radical formation and lipid peroxidation resulting in mitochondrial, lysosomal and sarcolemmal membrane damage. In the thyroid gland, this affects the production of thyroid hormones and manifests as varying degrees of primary hypothyroidism. Hence, it is postulated that higher serum ferritin levels predispose to a greater risk of developing endocrine-pathies like hypothyroidism. It has been suggested that thyroid dysfunction may be reversible by intensive chelation. Apart from iron overload, other factors responsible for organ damage have been previously pointed out including anemia and chronic hypoxia that may potentiate the toxicity of iron deposition in endocrines [16]. Also, viral infections as well as individual susceptibility have been implicated in causing endocrine dysfunction.

Malik et al [9] have reported primary hypothyroidism in $18(25.7 \%)$ out of 70 patients. Of these, 17 had normal T4 levels with elevated TSH levels consistent with a diagnosis of Subclinical hypothyroidism whereas only one patient showed a decreased T4 level with elevated TSH (overt hypothyroidism). Frequency of hypothyroidism was associated with increased serum ferritin levels. Primary hypothyroidism occurs in a significant proportion of thalassemia major patients in the absence of obvious clinical signs of hypothyroidism. Our results showed no association between Serum ferritin level and the frequency of hypothyroidism among studied patients; this finding is in agreement with results of studies elsewhere [17].

The absence of the relationship between ferritin and hypothyroidism may be explained by suggesting that the damage of endocrine glands caused by chronic hypoxia is more pronounced than that caused by hemosiderosis as a consequence of the collapse of iron [7].

\section{Conclusion}

Subclinical hypothyroidism occurs in a significant proportion of thalassemia major patients in the absence of obvious clinical signs of hypothyroidism. Regular follow-up for early detection and timely treatment of such complications could improve the quality of life of these patients.

Acknowledgment- The authors acknowledge the colleagues in the Department of Biochemistry, Medical College, Kolkata.

Funding: Nil, Conflict of interest: None initiated, Permission from IRB: Yes

\section{References}

1. Satwani H, Raza J, Alam M, Kidwai A. Endocrine Complications in Thalassaemias: Frequency and Association with Serum Ferritin Levels. Pak Paediat Assoc J 2005; 29: 113-9.

2. Lokeshwar MR. Late Hony. Surg. Cmde. Dr. Shantilal C. Sheth oration presentation during PEDICON 2006, Delhi, January 6th, 2006. Progress in 
the management of thalassemia. Indian Pediatr. 2006 Jun;43(6):503-6.

3. Shamshirsaz AA, Bekheirnia MR, Kamgar M, Pourzahedgilani N, Bouzari N, Habibzadeh M, Hashemi R, Shamshirsaz AA, Aghakhani S, Homayoun H, Larijani B. Metabolic and endocrinologic complications in beta-thalassemia major: a multicenter study in Tehran. BMC Endocr Disord. 2003 Aug 12;3(1):4.

4. Abdelrazik N, Ghanem H. Failure of puberty in Egyptian beta thalassemic patients: Experience in north east region - Dakahlia province. Hematology. 2007 Oct; 12 (5):449-56.

5. Al-Rimawi HS, Jallad MF, Amarin ZO, Al Sakaan R. Pubertal evaluation of adolescent boys with betathalassemia major and delayed puberty. Fertil Steril. 2006; 86:886-90.

6. Najafipour F, Aliasgarzadeh A, Aghamohamadzadeh $\mathrm{N}$, et al. A cross-sectional study of metabolic and endocrine complications in beta-thalassemia major. Ann Saudi Med. 2008; 28:361-6.

7. GulatiR, BhatiaV, AgarwalSS. Early onset of endocrine abnormalities in beta-thalassemia major in a developing country. J Pediatr Endocrinol Metab 2000; 13 (6):651-6.

8. Dodeigne $\mathrm{C}$, Thunus L, Lejeune R. Chemiluminescence as diagnostic tool. A review, Talanta 2000;51(3):415-39.

9. Malik SA, Syed S, Ahmed N.: Frequency of hypothyroidism in patients of beta-thalassemia. Pak I Med Assoc. 2010; 60(1): 17- 29.
10. Grundy R, Woods K, Savage M, et al.. Relationship of endocrinopathy to iron chelation status in young patients with thalassemia major. Arch Dis Child 1994; 71:128-32.

11. Gathwala G, Das K, Agrawal N. Thyroid hormone profile in Beta thalassemia major children. Indian $\mathbf{J}$ Pediatr 2009; 4(2):20-9.

12. Moaddab M, Hashemipour, M, Naderi M. The prevalence of endocrine complications in patients with thalassemia major. Eur Cong Endocrinol 2008; 16:578586.

13. Sharma $\mathrm{S}$ and Aggarwal R. Evaluation of thyroid hormones in Beta-thalassemic children of north India. UJMDS 2014; 2 (1):39-42.

14. Jain M, Sinha RS, Chellani H, Anand NK. Assessment of thyroid functions and its role in body growth in thalassemia major. Indian Pediatr. 1995 Feb;32(2):213-9.

15. Costin G, Kogut MD, Hyman CB, Ortega JA. Endocrine abnormalities in thalassemia major. Am J Dis Child. 1979; 133(5):497-502.

16. Magro S, Puzzonia $\mathrm{P}$, Consarino $\mathrm{C}$ et al. Hypothyroidism in patients with thalassemia syndromes. Acta Haematol. 1990; 84(2):72-6.

17. Khider NA, Hussein FM. Assessment of thyroid function among transfusion dependant thalassemics in Erbil. Middle East journal of family medicine Jan2014; 12(1):5-13.

\section{How to cite this article?}

Kundu D, Ray D, Ghosh S, Chowdhury DG, Dutta S, Dhar A. Relation of elevated serum ferritin levels to hypothyroidism in children with beta-thalassemia major. Int J Med Res Rev 2016;4(12):2120-2124.doi:10.17511 /ijmrr. 2016.i12.07. 